\title{
Light extraction and optical loss mechanisms in organic light-emitting diodes
}

\author{
Stefan Nowy, Nils A. Reinke+ ${ }^{+}$Jörg Frischeisen, and Wolfgang Brütting * \\ Experimental Physics IV, University of Augsburg, 86135 Augsburg, Germany
}

\begin{abstract}
The internal quantum efficiency of organic light-emitting diodes (OLEDs) can reach values close to $100 \%$ if phosphorescent emitters to harvest triplet excitons are used, however the fraction of light that is actually leaving the device is considerably less. Loss mechanisms are for example waveguiding in the organic layers and the substrate as well as the excitation of surface plasmon polaritons at metallic electrodes. In this work we use numerical simulations to identify and quantify different loss mechanisms. Changing various simulation parameters, for example layer thicknesses, enables us to study their influence on the fraction of light leaving the OLED. With these simulations we therefore can enhance the light output of the OLED stack.

We present simulations of bottom-emitting OLEDs based on the green emitter tris-(8-hydroxyquinoline) aluminum $\left(\mathrm{Alq}_{3}\right)$ with transparent indium tin oxide anode and a metallic cathode, as well as microcavity devices with two metallic electrodes. The results of the simulations are compared with experimental data on the angular dependent emission spectra and published efficiency data.
\end{abstract}

Keywords: organic light-emitting diode, dipole model, light extraction, angular emission spectrum

\section{EXPERIMENTAL METHODS}

\subsection{Sample structure and fabrication}

The substrates for the bottom-emitting organic light-emitting diodes (OLEDs) consist of glass with a prestructured, $140 \mathrm{~nm}$ thick indium tin oxide (ITO) layer (Merck). A $30 \mathrm{~nm}$ thin layer of poly(3,4)-ethylendioxythiophene doped with poly(styrene sulfonate) (PEDOT:PSS) is spincast onto the substrate and dried on a hot plate. Devices with ITO anode are referred to as ITO OLED (fig. 1). For microcavity OLEDs a thin metal layer is deposited onto a glass substrate; a $30 \mathrm{~nm} \mathrm{Ag} \mathrm{layer} \mathrm{is} \mathrm{used} \mathrm{in} \mathrm{the} \mathrm{device} \mathrm{shown} \mathrm{in} \mathrm{this} \mathrm{work} \mathrm{(fig.} \mathrm{2).} \mathrm{Commonly}$ for both device types all following organic and metal layers are deposited through shadow masks in a high vacuum chamber (base pressure $<3 \cdot 10^{-7} \mathrm{mbar}$ ) without breaking the vacuum. Organic materials are deposited using effusion cells. As hole transporter N,N'-diphenyl-N,N'-bis(3-methylphenyl)-1,1'-biphenyl-4,4-diamine (TPD) with a thickness of $80 \mathrm{~nm}$ is used. The emitter is tris-(8-hydroxyquinoline) aluminum $\left(\mathrm{Alq}_{3}\right)$ with a thickness of $80 \mathrm{~nm}$, which has a photoluminescence (PL) peak emission of about $535 \mathrm{~nm}$ (fig. 3). As cathode, deposited by thermal evaporation, aluminum on top of calcium is used.

After evaporation current-voltage (I-V) and luminance-voltage (L-V) characteristics of the OLEDs are recorded simultaneously in a glovebox system under dry $\mathrm{N}_{2}$ atmosphere to find the working point of the devices. Before taking the samples out to ambient air they are encapsulated with a glass slide and epoxy glue.

* Corresponding author: wolfgang.bruetting@physik.uni-augsburg.de

$+\quad$ Present address: Institute for Computational Physics, Zurich University of Applied Sciences, PO Box 805, 8401 Winterthur, Switzerland

Organic Optoelectronics and Photonics III, edited by Paul L. Heremans, Michele Muccini, Eric A. Meulenkamp, Proc. of SPIE Vol. 6999, 69992V, (2008) - 0277-786X/08/\$18 $\cdot$ doi: 10.1117/12.780525 


\begin{tabular}{|l|r|}
\hline $\mathrm{Al}$ & $100 \mathrm{~nm}$ \\
\hline $\mathrm{Ca}$ & $15 \mathrm{~nm}$ \\
\hline $\mathrm{Alq}_{3}$ & $80 \mathrm{~nm}$ \\
\hline $\mathrm{TPD}$ & $80 \mathrm{~nm}$ \\
\hline PEDOT:PSS & $30 \mathrm{~nm}$ \\
\hline ITO & $140 \mathrm{~nm}$ \\
\hline glass \\
\hline
\end{tabular}

Figure 1. OLED stack with transparent indium tin oxide (ITO) anode

\begin{tabular}{|ll|}
\hline $\mathrm{Al}$ & $80 \mathrm{~nm}$ \\
\hline $\mathrm{Ca}$ & $40 \mathrm{~nm}$ \\
\hline $\mathrm{Alq}_{3}$ & $80 \mathrm{~nm}$ \\
\hline $\mathrm{TPD}$ & $80 \mathrm{~nm}$ \\
\hline $\mathrm{Ag}$ & $30 \mathrm{~nm}$ \\
\hline glass \\
\hline
\end{tabular}

Figure 2. OLED stack with Ag anode (microcavity OLED)

\subsection{Angular emission spectrum of OLEDs}

One of the advantages of OLEDs as large area light-emitting devices is that the color when looking at the device under different angles is changing only very little. However tailoring this angular dependence to achieve better color stability over the viewing angle is a common goal. Thus one aim of our simulations is to predict the angular dependent emission spectra of OLEDs.

The angular emission spectrum of an OLED is recorded with a calibrated CCD spectrometer. The OLED is placed on a computer controlled rotary stage. Light emitted at a given angle is focused by a collimator lens and coupled into an optical fiber, which leads to the spectrometer. Furthermore a semi-circle prism can be attached to the OLED's glass substrate by applying an optical gel. Compared to the spectrum without the prism this additionally allows the study of light which normally is guided in the substrate. Without the prism this light can not leave the substrate directly due to total internal reflection caused by the difference in the refractive index of the glass/air interface $\left(n_{\text {glass }} \approx 1.51, n_{\text {air }}=1\right)$.

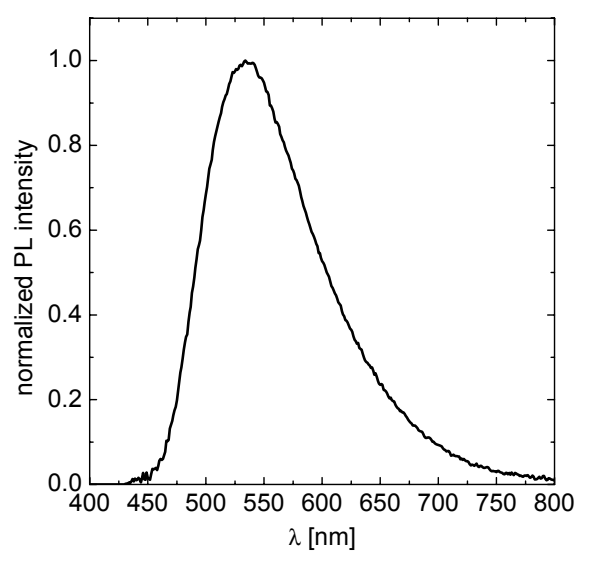

Figure 3. Photoluminescence (PL) spectrum of $\mathrm{Alq}_{3}$
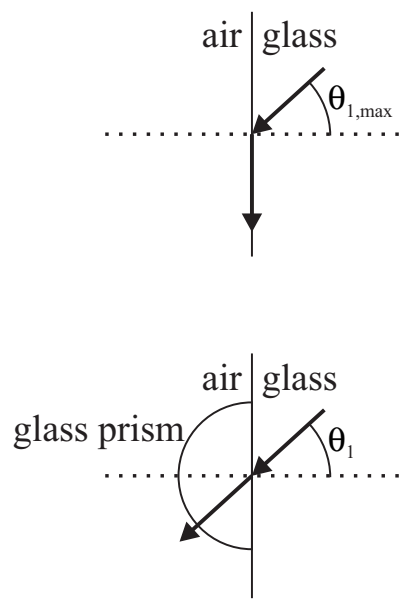

Figure 4. Maximum internal angle for glass/air interface $\theta_{1, \max } \approx 42^{\circ}$ (top) and the case for attached semi-circle prism (bottom)

\section{SIMULATION METHOD}

The simulations presented in this work are based on a model of emissive dipoles. The dipoles, embedded in the multilayer stack of an OLED, are treated as forced damped harmonic oscillators ${ }^{1}$

$$
\frac{\mathrm{d}^{2} p}{\mathrm{~d} t^{2}}+\omega_{0}^{2} p=\frac{e^{2}}{m} E_{\mathrm{r}}-b_{0} \frac{\mathrm{d} p}{\mathrm{~d} t},
$$


where $p$ is the electric dipole moment, $\omega_{0}$ is the resonant angular frequency in the absence of damping, $m$ is the effective mass, $e$ the electric charge, $E_{\mathrm{r}}$ is the reflected field at the dipole position and $b_{0}$ is the damping constant due to the finite radiative lifetime of the emitter. Layers in this multilayer stack are treated as infinite in two dimension, having a certain thickness in the third direction. The interface between two layers is assumed sharp and without roughness. Using a transfer matrix method the Fresnel coefficients are calculated by taking the optical constants and thicknesses of the different layers of the OLED stack into account, as well as the emission position of the dipoles within the OLED, which is assumed to be sharp as well. Then the Helmholtz equation is solved by a development into plane waves using the previously obtained coefficients. Finally the orientation of the dipoles (parallel or perpendicular to the substrate, weighted for isotropic dipole orientation) and the emission spectrum of the emitter is taken into account. The quantum efficiency q is a variable in the simulation, which also handles any non-radiative decay of the emitter dipoles. For $\mathrm{Alq}_{3}$ it is assumed to be $q=0.2 \cdot \frac{1}{4}=0.05$, i.e. the photoluminescence quantum efficiency times the singlet/triplet ratio, as it is a fluorescent emitter. The simulation results will be compared to a system with $q=1.0$, i.e. an OLED comprising a phosphorescent emitter with $100 \%$ internal quantum efficiency.

This simulation method is similar to the work published by the group of W. L. Barnes,${ }^{1-3}$ however our simulation includes the transmission (and therefore absorption losses) of light through the different layers of the OLED stack. As a result of the simulation we obtain a polychromatic power dissipation spectrum depending on the in-plane wave vector $k_{\mathrm{x}}$. The concept of in-plane wave vectors is very useful: it not only describes light traveling under a certain angle in a layer of the OLED, it also is a constant for all layers (fig. 5). Furthermore high values of this vector $\left(k_{\mathrm{x}}>\frac{2 \pi}{\lambda} \cdot n_{\text {glass }}(\lambda)\right)$ represent non-radiative modes (waveguided modes and surface plasmon polaritons (SPP) which are near-field phenomena that are not described by ordinary plane waves for far field radiation). The power dissipation spectra therefore can be split up into three regions (figure 6), where we can identify and quantify the extracted fraction of light which can leave the OLED directly (region 1), the fraction of light which is emitted to the substrate and can not leave the device without outcoupling structures (region 2), and waveguided modes, as well as SPPs (both in region 3).

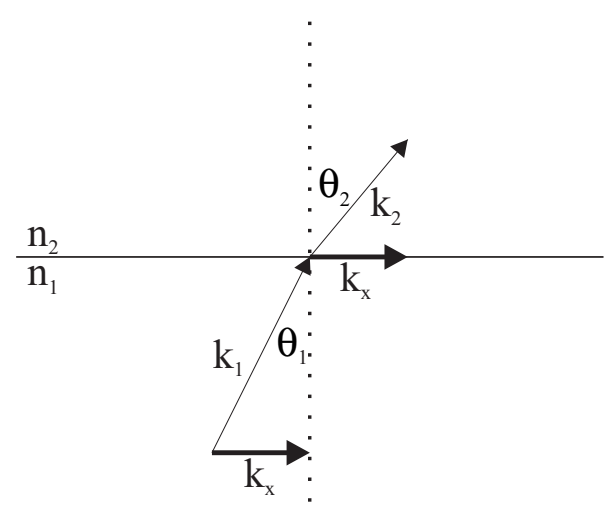

Figure 5. In-plane wave vector $k_{\mathrm{x}}$ is constant for light crossing the interface of medium 1 and medium 2 (Snell's law)

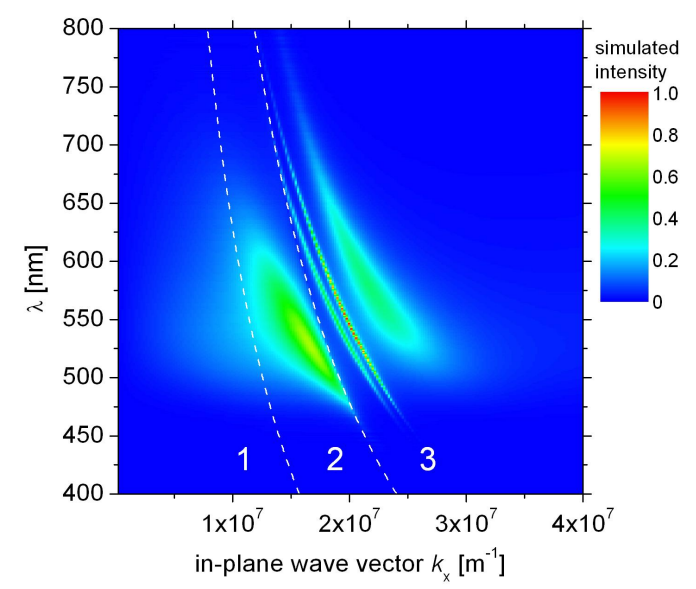

Figure 6. Typical power dissipation spectrum of an ITO OLED: 1) light emitted directly from the OLED, 2) light trapped inside substrate, 3 ) non-radiative modes

The identification of possible transverse electric (TE) and transverse magnetic (TM) modes uses a transfer matrix method, which is based on a basic three-layer waveguide calculation. ${ }^{4}$ After considering the values of the in-plane wave vector $k_{\mathrm{x}}$ the waveguided modes and the plasmons (which have TM polarization) can be clearly identified (figure 7). A cross section at $\lambda=525 \mathrm{~nm}$ can be seen in figure 8 . With these findings, integrating over the different areas in the power dissipation spectrum leads to the amount of power coupled into the different 
modes (light emitted directly: $1.1 \%$, light emitted to the substrate: $1.6 \%$, waveguided modes: $0.6 \%$, plasmons: $2.6 \%$, all other losses: $94.1 \%$ ). Compared to the calculations from different groups in the literature ${ }^{5,6}$ we can confirm their basic findings, which strengthens the confidence for our simulation tool. There are, however, several other possibilities to simulate the emission spectra of OLEDs, that do not grant access to the non-radiative modes and often are limited to specific stack types like microcavities. ${ }^{7-9}$

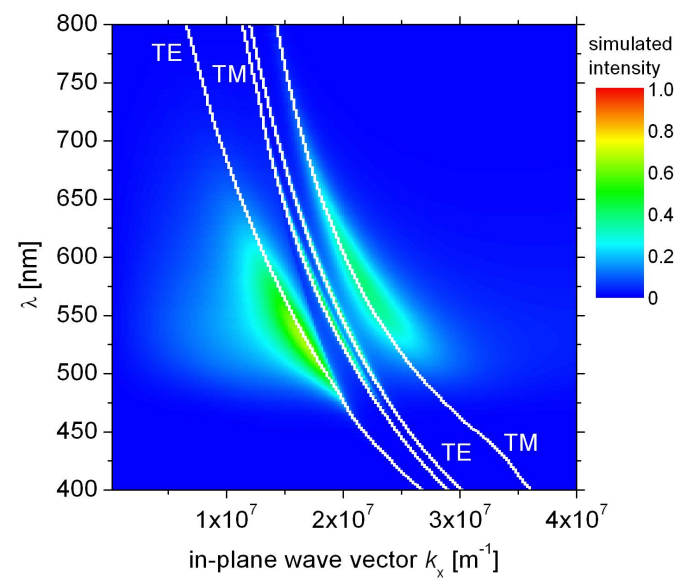

Figure 7. Same power dissipation spectrum as fig. 6: identification of TE and TM modes. Waveguided modes and plasmons are located in region 3

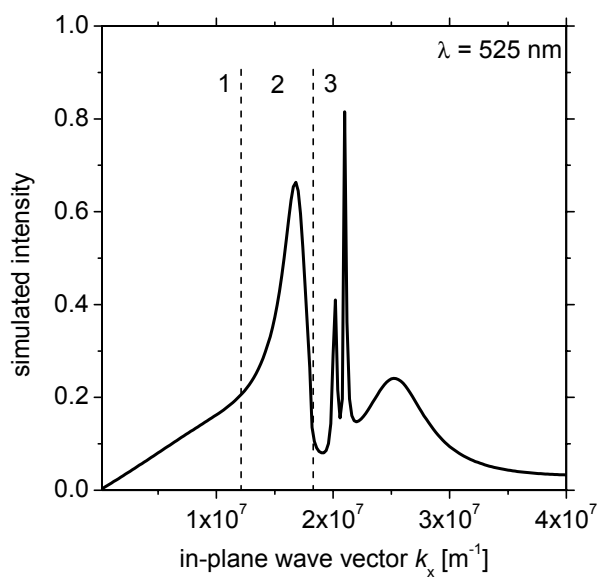

Figure 8. Cross section of fig. 7 at $\lambda=525 \mathrm{~nm}$ : 1) light emitted directly, 2) light trapped inside substrate, 3) two sharp waveguided modes and an SPP

\section{COMPARISON OF EXPERIMENTAL AND SIMULATED OLED EMISSION SPECTRA}

To confirm the outcome of the simulations they are compared to the experimental angular emission spectra of the devices. Therefore the angle of emission $\theta$ is calculated from the in-plane wave vector $k_{x}$.

$$
\theta\left(k_{\mathrm{x}}, \lambda\right)=\arcsin \frac{k_{\mathrm{x}}}{k(n(\lambda), \lambda)}=\arcsin \left(\frac{k_{\mathrm{x}}}{2 \pi n(\lambda)} \lambda\right)
$$

$\lambda$ is the wavelength of the light, $n(\lambda)$ is the refractive index either of air for light emitted directly from the device $\left(n(\lambda)=n_{\text {air }}\right)$ or it is the refractive index of the glass substrate for light trapped inside the substrate $\left(n(\lambda)=n_{\text {glass }}(\lambda)\right)$. The emission spectra of the ITO OLED (already presented in figure 1 ) are shown in figure 9 with distinction of s- and p-polarized light emission.

These spectra are in very good agreement concerning the angular characteristics. The spectral pattern is slightly broadened at high wavelengths in the simulation; this might be due to a too broad PL spectrum of $\mathrm{Alq}_{3}$ used in the simulations. Both simulation and experiment show the maximum emission perpendicular to the substrate $\left(\theta=0^{\circ}\right)$. The decrease in the emission intensity at higher angles $\theta$ follows Lambert's cosine law. Since the emission pattern is changing very little with the angle $\theta$, the device's color stability is very good. Light emitted from this ITO device is mainly unpolarized, since the s-polarized and p-polarized spectra differ only very little. Figure 10 shows the same device with a semi-circle prism attached to the glass substrate. Here again the simulated spectra are in good agreement with the experiment. Comparisons of these results to the plain OLED (fig. 9) show that in the latter a considerable amount of radiation is trapped inside the glass substrate. As the intensity of light emitted normal to the substrate is the same for both cases (with and without prism), there is actually a big portion of light trapped at high angles: the peak intensity at about $\theta \approx 65^{\circ}$ is almost two times 


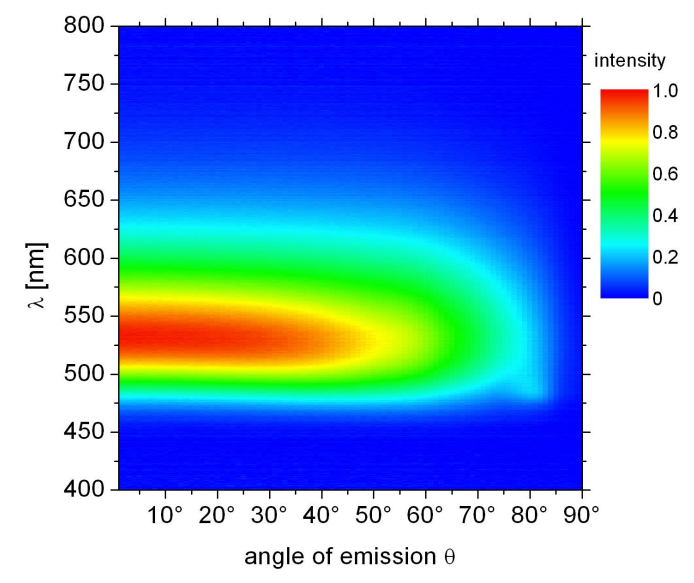

(a) s-pol., experiment

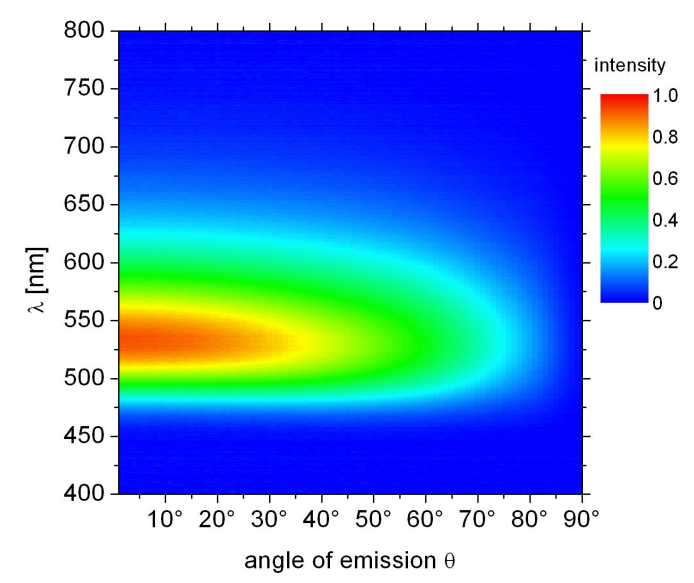

(c) p-pol., experiment

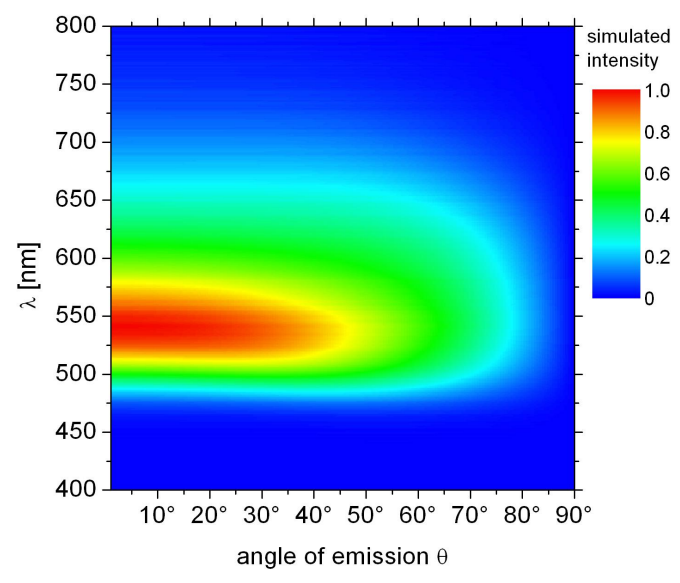

(b) s-pol., simulation

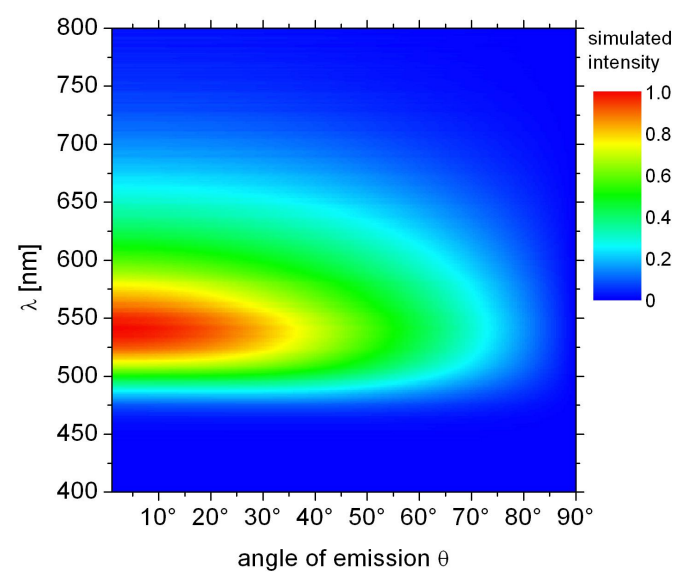

(d) p-pol., simulation

Figure 9. s- and p-polarized angular emission spectra of the ITO OLED (for details see text) 
higher than the peak intensity of the perpendicular emission. Please note that the figures are normalized with respect to the highest intensity obtained in each of them, except that corresponding s- and p-polarized spectra are normalized with the same factor. The difference in s- and p-polarized emission shows that the trapped light is s-polarized. So after attaching an outcoupling structure to the OLED substrate an s-polarized leaky mode is emitted, having a slight dispersion in the wavelength.

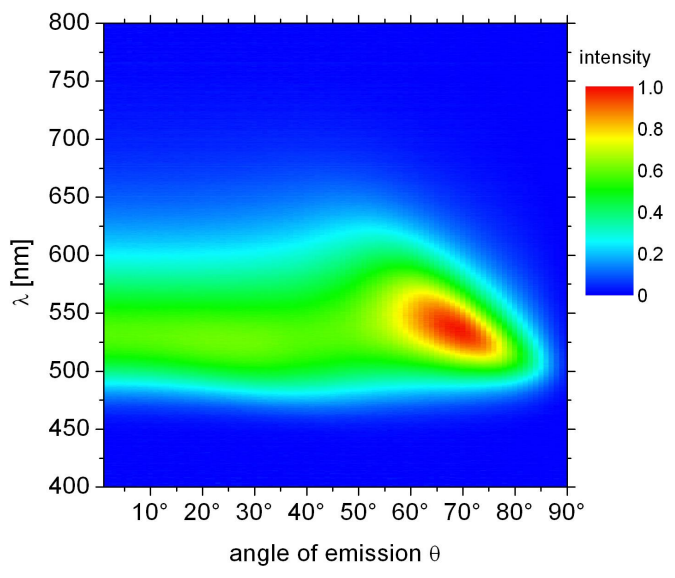

(a) s-pol., experiment

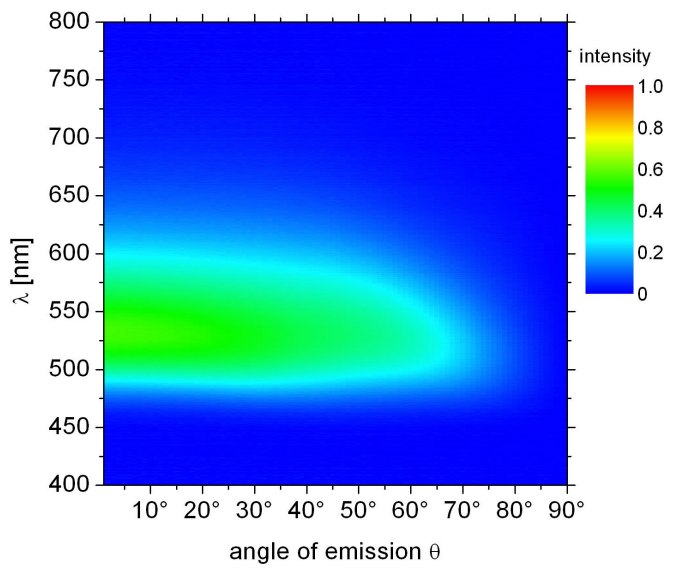

(c) p-pol., experiment

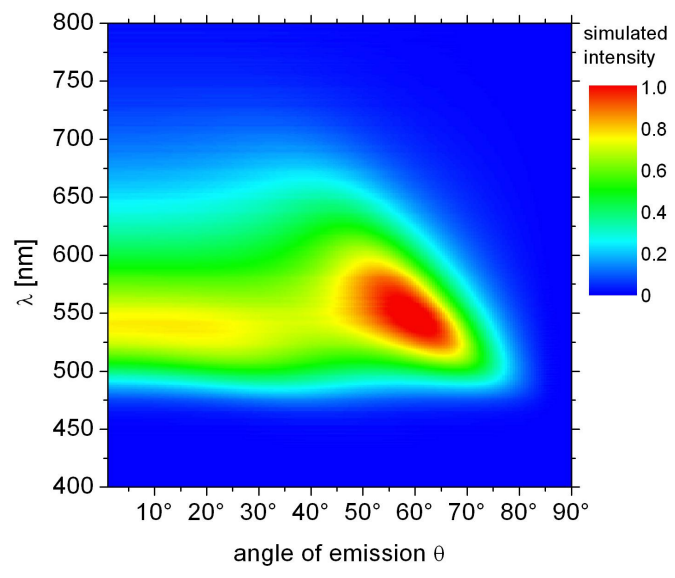

(b) s-pol., simulation

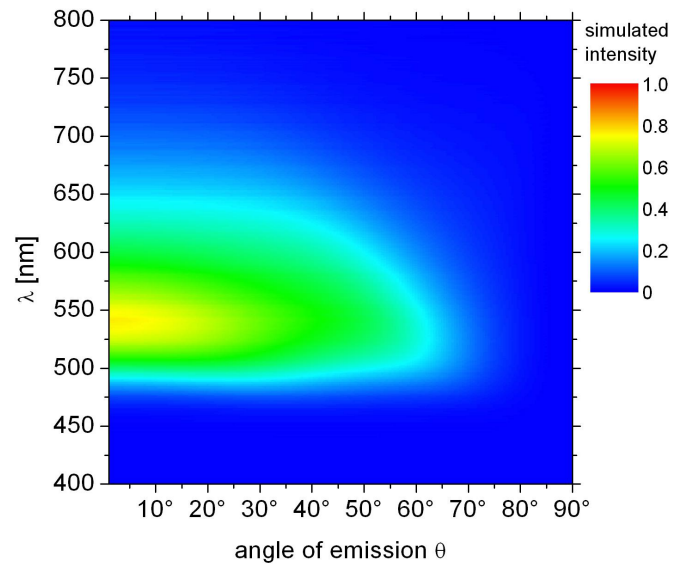

(d) p-pol., simulation

Figure 10. s- and p-polarized angular emission spectra of the ITO OLED with attached semi-circle prism (for details see text)

In figure 11 the spectra for a microcavity device, presented earlier in figure 2, are shown. The features of the angular emission of the microcavity OLED are again well represented by the simulation. In contrast to the ITO OLED the emission spectrum of this microcavity device is now strongly dependent on the emission angle $\theta$. Also the angular emission spectra are changing drastically over a wide wavelength range. This is even more pronounced if the semi-circle prism is attached to the microcavity device (not shown here). This kind of emission spectrum is due to interference effects of light traveling in the "waveguide" between the two metallic electrodes, resulting in an s-polarized leaky mode. ${ }^{9}$ We would like to note that we also verified the simulations 
with experimental data for other microcavity OLEDs with different anode metals, but this is not shown here.

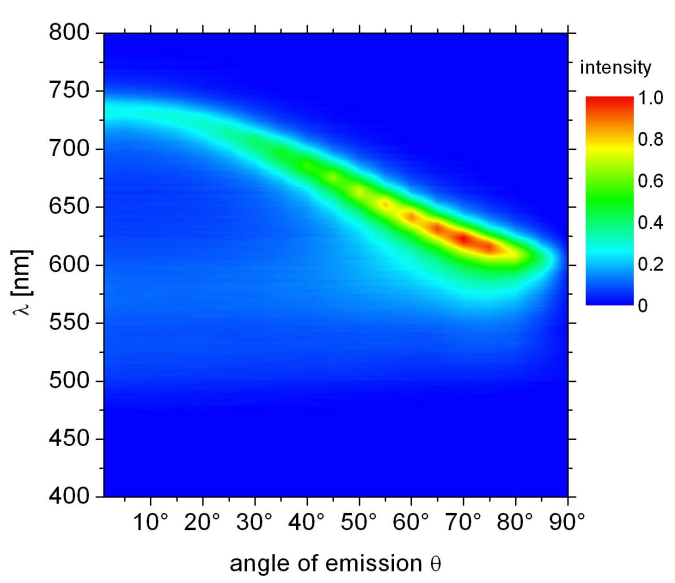

(a) s-pol., experiment

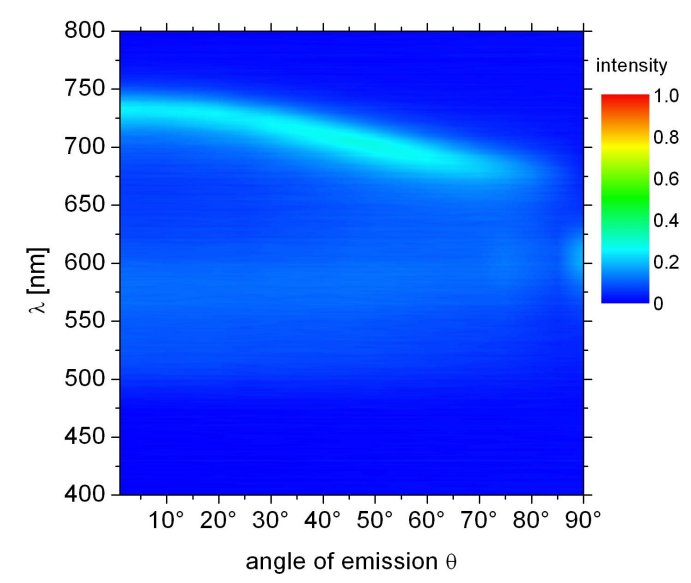

(c) p-pol., experiment

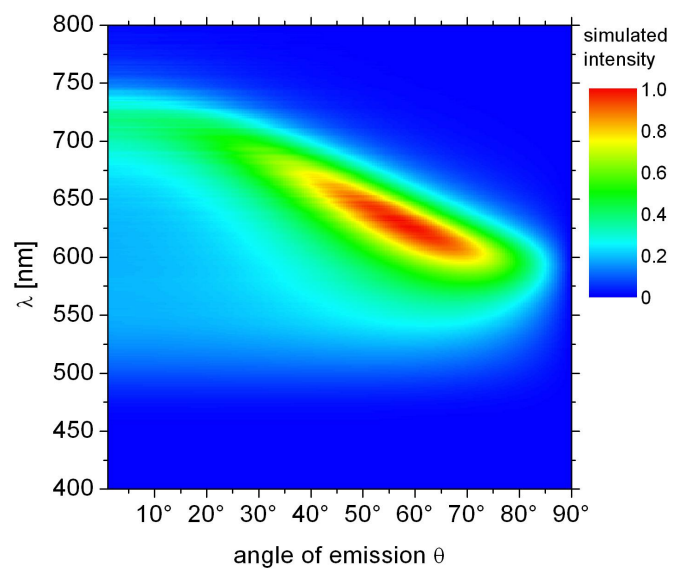

(b) s-pol., simulation

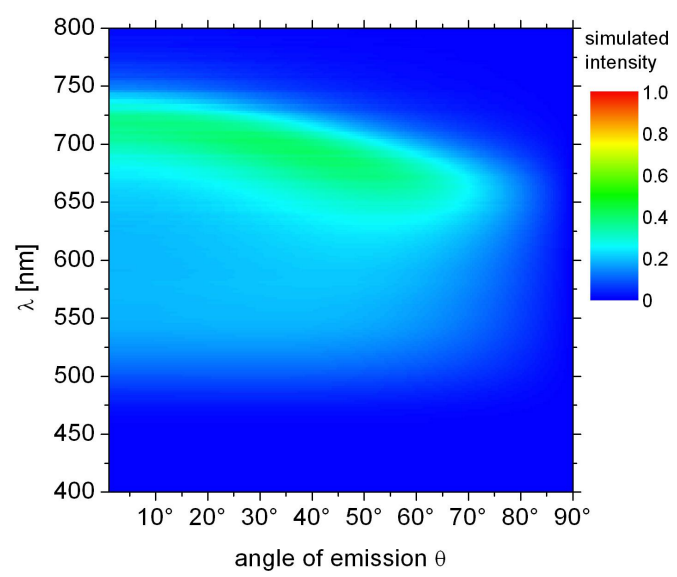

(d) p-pol., simulation

Figure 11. s- and p-polarized angular emission spectra of the microcavity OLED without prism (for details see text)

\section{ENHANCING LIGHT OUTPUT}

In the previous sections we have seen that our simulations are in good agreement with experiments on angular dependent emission spectra of OLEDs. Now we can use optical simulations to find optimum conditions for the multilayer OLED stack and therefore enhance the light output of OLEDs. A strong influence on the amount of energy coupled into waveguided modes comes from the thickness of the various layers and the dipole layer position within the OLED stack. For the plasmonic modes the distance of the dipole layer to metallic electrodes is of great importance. We present variations of the ITO OLED introduced in section 1 . The device structure of $140 \mathrm{~nm}$ ITO, $30 \mathrm{~nm}$ PEDOT:PSS, $80 \mathrm{~nm}$ TPD, $80 \mathrm{~nm} \mathrm{Alq}_{3}$, $15 \mathrm{~nm} \mathrm{Ca}$, and $100 \mathrm{~nm} \mathrm{Al} \mathrm{(figure} \mathrm{1)} \mathrm{is} \mathrm{the} \mathrm{basis}$ for all following simulations unless otherwise noted. We note that this well-known structure, though not being particularly efficient, still serves as a model system for OLEDs with a single emitting material enclosed by a hole 
and electron conducting compartment. This does also include phosphorescent emitters where in the simulations basically only the internal quantum efficiency has to be increased to values close to unity. Additionally we have verified published data on light extraction from phosphorescent OLEDs with our simulation routine. ${ }^{5,6}$

In figure 12 the dipole layer position is shifted successively from the TPD/Alq ${ }_{3}$-interface to the $\mathrm{Ca} / \mathrm{Al}$ cathode* What can be seen here is that the fraction of outcoupled light and light emitted to the substrate is strongly decreased when the dipole layer is moving closer to the cathode. This is due to the strongly increasing plasmonic mode which gains power on shortening the distance of the dipole layer to the metallic cathode. To achieve high efficiencies for this OLED device it is best, if the dipole layer is close to TPD/Alq ${ }_{3}$-interface, which for this stack is generally assumed to be the case. All further simulations shown in this work make use of this assumption, locating the dipole layer at the $\mathrm{TPD} / \mathrm{Alq}_{3}$-interface. The waveguided modes, by contrast, are only influenced a little by the variation of the dipole layer position, since no layer thickness in the device is changing. These modes will be addressed in the next simulations.

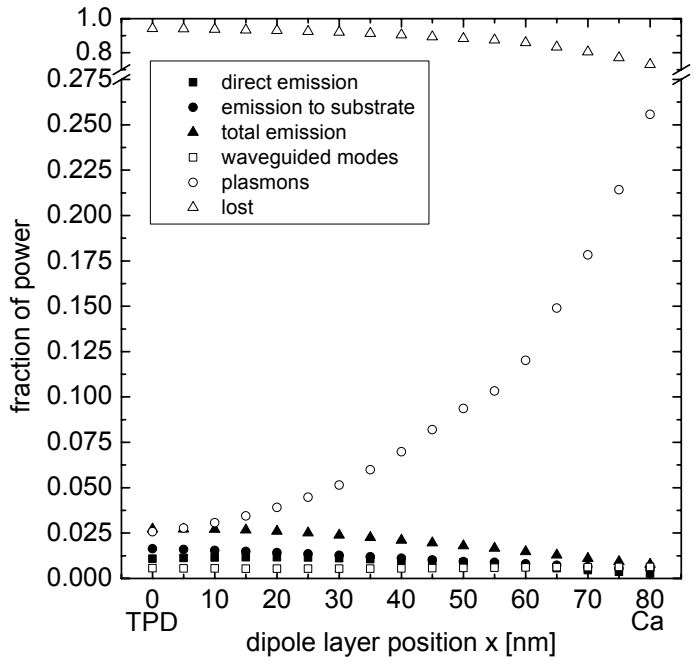

(a) overview

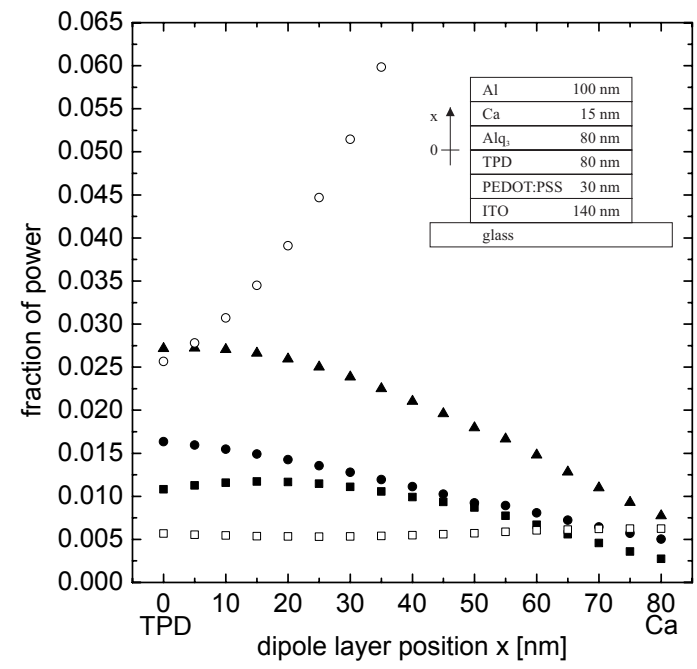

(b) zoom of (a)

Figure 12. Variation of the dipole layer position within the $\mathrm{Alq}_{3}$ layer from the TPD/Alq ${ }_{3}$-interface to the $\mathrm{Alq} \mathrm{q}_{3} / \mathrm{Ca}$ interface, internal quantum efficiency $q=0.05$

Changing the thickness of one organic layer shows the influence of the waveguided modes on the emission of light, as can be seen in figure 13, where the TPD layer thickness is varied. The dipole layer has a fixed distance from the metallic cathode, resulting in an almost constant plasmon contribution. From an optical point of view the fraction of light directly coupled out has its maximum at small TPD layer thicknesses, whereas the amount of light trapped inside the substrate has its maximum at a layer thickness where the waveguided modes have a minimum. This shows that the coupling to waveguided modes can be reduced to the benefit of light which can leave the device through outcoupling structures; however the total amount of light (direct emission and emission to the substrate added together) would, from an optical point of view, suggest a preferably very thin TPD layer. After these results one could imagine that either the TPD layer should be avoided for optical reasons or that a shorter distance of the dipole layer to the anode could improve the fraction of light emitted from the OLED. For this reason we applied changes to the anode side of the ITO OLED stack: the PEDOT:PSS layer is omitted and the ITO thickness is reduced to $50 \mathrm{~nm}$; the TPD layer thickness is still subject of variation. The results shown in figure 14 show a clear maximum (about 1.3\%) for the direct emission at about $90 \mathrm{~nm}$ TPD layer thickness. Together with the previous simulation (figure 13) this leads to the conclusion that the TPD layer alone does not have a negative influence on the optical properties of the $\mathrm{Alq}_{3}$-OLED stack, but that the distance of the dipole

${ }^{*}$ Please note that the total emission in all following figures is the sum of the direct emission and the emission to substrate. 
layer to the glass substrate is of importance here.

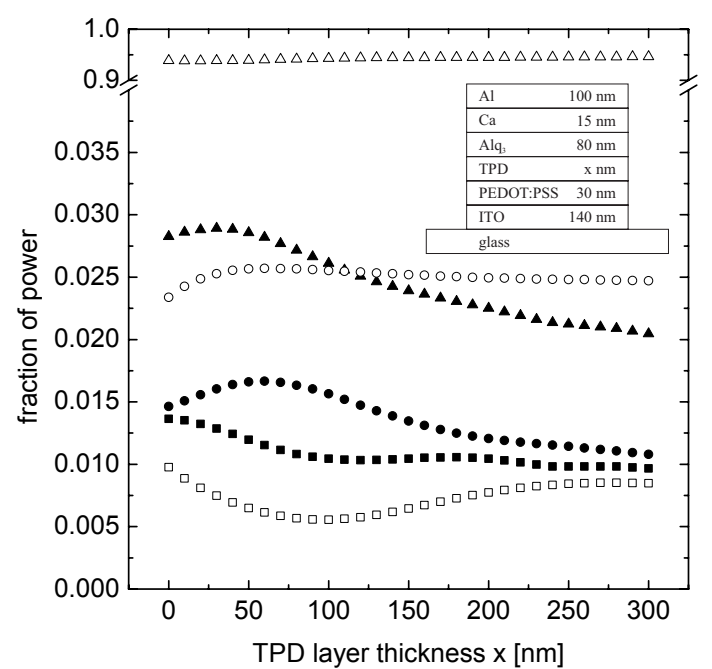

Figure 13. TPD layer thickness variation, internal quantum efficiency $q=0.05$ (symbols as in fig. 12)

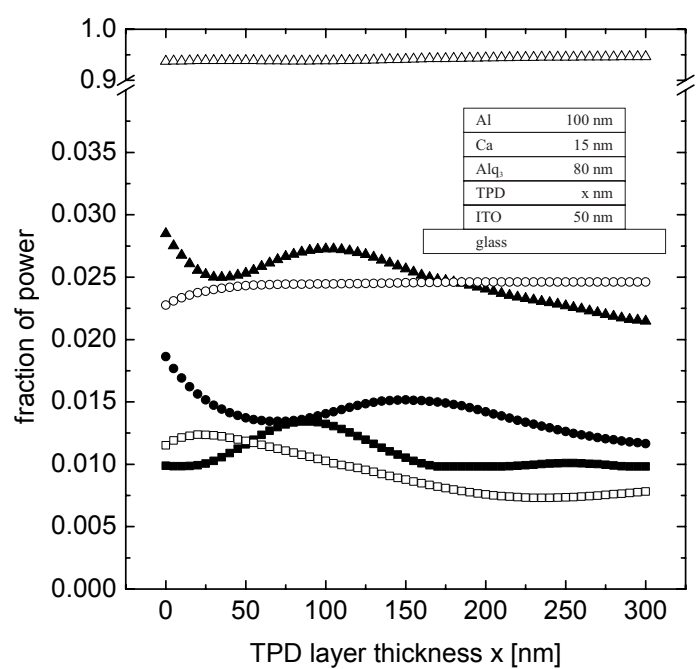

Figure 14. Modified ITO OLED with $50 \mathrm{~nm}$ ITO, no PEDOT:PSS layer: TPD layer thickness variation, internal quantum efficiency $q=0.05$ (symbols as in fig. 12)

The next simulations show the variation of the $\mathrm{Alq}_{3}$ layer thickness (figure 15). Here not only a layer thickness is changing, influencing the waveguided modes, but also the dipole layer position with respect to the cathode is changing, resulting in a variation of the plasmon contribution. As the dipole layer is moved further away from the cathode the coupling to the SPP is reduced drastically. For the direct emission there exist several local maxima and minima; the first maximum at around $60 \mathrm{~nm} \mathrm{Alq}$ layer thickness shows the highest fraction of outcoupled light (about 1.2\%). This thickness (which at the same time is the distance of the dipole layer to the cathode) and also the value of the maximum external quantum efficiency is consistent with the optimal thickness of Alq 3 found in experimental work. ${ }^{10,11}$ However if outcoupling structures are attached to the OLED glass substrate the maximum fraction of total outcoupled light (about 2.7\%) is achieved with an Alq layer thickness of about $80 \mathrm{~nm}$.

As the thickness of $\mathrm{Alq}_{3}$ increases further a second maximum in the direct emission appears, which is slightly lower in value (about 1.0\%) than the first maximum. Calculations where the quantum efficiency is assumed to be $q=1.0$ show that this second maximum can be even higher than the first one (figure 16). This effect has also been reported in literature. ${ }^{6}$ OLED stacks with high quantum efficiency emitters therefore would benefit from an increased layer thickness on the cathode side of the emitter, as this increases the distance of the dipoles to the cathode, thus resulting in a smaller SPP contribution.

It should be noted that the optimizations of ITO OLEDs presented here consider only the optical performance of the devices. Altering layer thicknesses will change the electrical performance, which has to be optimized as well. In this context, doped electron and hole transport layers will be important tools. Optimization of both the optical and electrical performance may require a compromise to be made, however this is not addressed in this work.

\section{SUMMARY}

In conclusion the simulations presented here show that the optical performance of OLEDs depends on several factors. Clearly the layer thicknesses have an influence on the waveguided modes. The dipole layer has to be located at the right position, so that the waveguided modes are optimal for the fraction of light emitted from the device and the fraction coupled to SPPs is minimized. The optimal dipole layer position also depends on the quantum efficiency of the material used as emitter. Therefore the internal quantum efficiency should be 


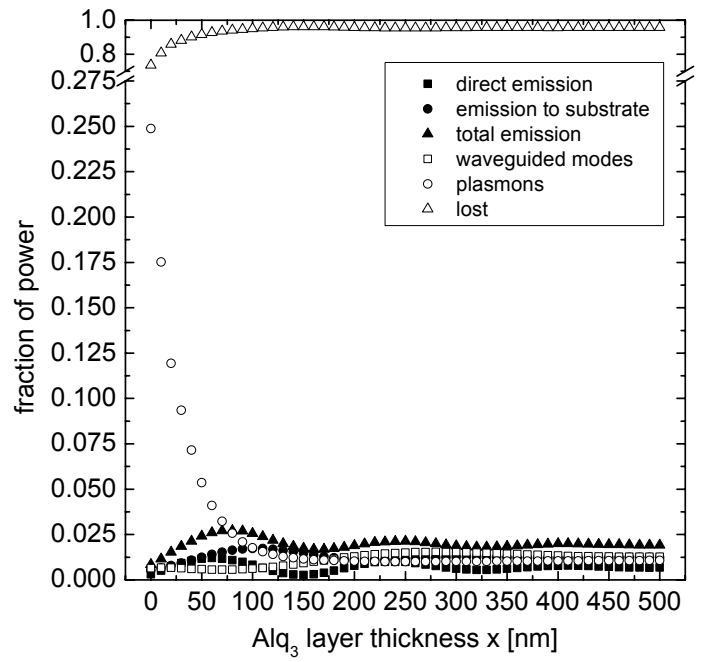

(a) overview

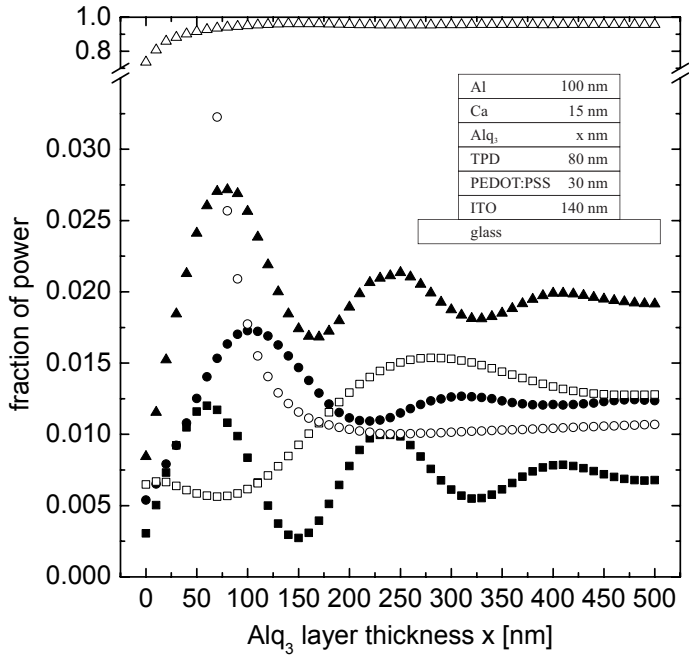

(b) zoom of (a)

Figure 15. $\mathrm{Alq}_{3}$ layer thickness variation, internal quantum efficiency $q=0.05$

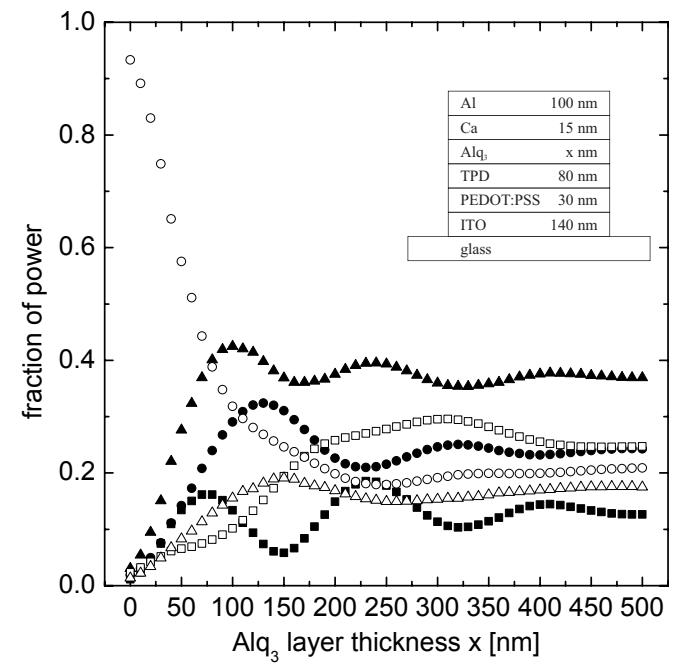

Figure 16. $\mathrm{Alq}_{3}$ layer thickness variation, internal quantum efficiency $q=1.0$ (symbols as in fig. 15) 
known for accurate layer thickness optimizations. Last, when optimizing the device it has to be considered if outcoupling structures will be used for the OLED, as the optimal layer thicknesses are slightly different to the ones for a plain OLED.

Nevertheless, it is evident from these results that even in an optimized structure (with $100 \%$ internal quantum efficiency) only some $40 \%$ of the radiation generated inside the OLED can actually be extracted, provided that all of the light guided as substrate modes could be coupled out using suitable techniques. Further enhancement of the external OLED efficiency will therefore require the development of methods to couple out radiation from waveguided modes or even surface plasmons.

\section{ACKNOWLEDGMENTS}

The authors would like to thank the German Federal Ministry of Education and Research (BMBF) for funding part of this work under contract FKZ 13N8995 (OPAL).

Furthermore we like to thank B. Krummacher from OSRAM Opto Semiconductors GmbH, Regensburg, Germany, for fruitful discussions and support.

\section{REFERENCES}

[1] Barnes, W. L., "Fluorescence near interfaces: the role of photonic mode density," Journal of Modern Optics 45(4), 661 - 699 (1998).

[2] Wasey, J. A. E. and Barnes, W. L., "Efficiency of spontaneous emission from planar microcavities," Journal of Modern Optics 47(4), 725 - 741 (2000).

[3] Smith, L. H., Wasey, J. A. E., Samuel, I. D. W., and Barnes, W. L., "Light out-coupling efficiencies of organic light-emitting diode structures and the effect of photoluminescence quantum yield," Advanced Functional Materials 15(11), 1839 - 1844 (2005).

[4] Kogelnik, H., "Theory of optical waveguides," in [Guided-Wave Optoelectronics], Tamir, T., ed., Springer (1990).

[5] Adachi, C., Baldo, M. A., Thompson, M. E., and Forrest, S. R., "Nearly 100\% internal phosphorescence efficiency in an organic light emitting device," Journal of Applied Physics 90(10), 5048 - 5051 (2001).

[6] Lin, C.-L., Cho, T.-Y., Chang, C.-H., and Wu, C.-C., "Enhancing light outcoupling of organic light-emitting devices by locating emitters around the second antinode of the reflective metal electrode," Applied Physics Letters 88, 081114 (2006).

[7] Neyts, K. A., "Simulation of light emission from thin-film microcavities," Journal of the Optical Society of America A 15(4), 962 - 971 (1998).

[8] Ruhstaller, B., Beierlein, T., Riel, H., Karg, S., Scott, J. C., and Riess, W., "Simulating electron and optical processes in multilayer organic light-emitting devices," IEEE Journal on Selected Topics in Quantum Electronics 9(3), 723 - 731 (2003).

[9] Reinke, N. A., Ackermann, C., and Brütting, W., "Light extraction via leaky modes in organic light emitting devices," Optics Communications 266, 191 - 197 (2006).

[10] Schmitz, C., Thelakkat, M., and Schmidt, H.-W., "A combinatorial study of the dependence of organic LED characteristics on layer thickness," Advanced Materials 11(10), 821 - 826 (1999).

[11] Beierlein, T. A., Combinatorial Methods for Organic Light-Emitting Materials and Devices, PhD thesis, University of Bayreuth, Germany (2003). 